\title{
Vogt - Koyanagi Harada Syndrome in a Pakistani Female
}

\author{
Rebecca $^{1}$, Murtaza Sameen Junejo ${ }^{2}$, Fahad Feroz Shaikh ${ }^{3}$, Nazir Ashraf Laghari ${ }^{4}$ \\ ${ }^{7-4}$ Department of Ophthalmology, Isra University Hospital, Hyderabad
}

\begin{abstract}
Vogt-Koyanagi-Harada syndrome is a rare multiorgan inflammatory disorder characterized by bilateral uveitis with serous retinal detachment and is often associated with headache, hearing loss, vitiligo, and poliosis. Here we present a case of 37 years old female who presented with chronic photophobia, redness and progressive decreased vision in both eyes for 5 years along with cutaneous and hearing symptoms. On Ocular examination her best-corrected visual acuity was 6/60 OD and perception of light OS, while the intraocular pressure was 16

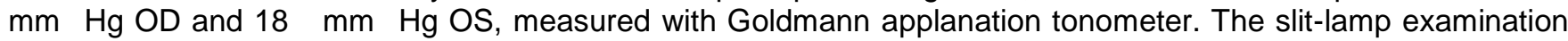
of right anterior segment showed diffuse Keratic precipitates along with diffuse iris atrophy and seclusion pupillae (360 degree). On detailed examination she was found to be a case of VKH syndrome. This case is presented to familiarize ophthalmologists and health care professionals about its findings and complications that are usually found in such patients.
\end{abstract}

Key Words: Uveitis, Poliosis, Vogt Koyanagi Harada, vitiligo.

How to Cite this Article: Rebecca, Junejo MS, Shaikh FF, Laghari NA. Vogt - Koyanagi Harada Syndrome in a Pakistani Female. Pak J Ophthalmol. 2020; 36 (4): 445-447.

Doi: https://doi.org/10.36351/pjo.v36i4.1120

\section{INTRODUCTION}

Vogt-Koyanagi Harada syndrome is a rare multi organ inflammatory disease characterized by uveitis and serous retinal detachment. Neurological and cutaneous associations have also been found in this condition, which includes headache, poliosis, hearing loss, and vitiligo. ${ }^{1}$ The etiology and pathogenesis of $\mathrm{VKH}$ syndrome remains unknown. A few studies have shown an association between HLA-DR4 and HLA$D R 53$ with VKH syndrome. In this granulomatous autoimmune disorder, melanocyte containing organs are highly affected. One out of three findings are necessary for the diagnostic criteria for $\mathrm{VKH}$

Correspondence: Murtaza Sameen Junejo

Department of Ophthalmology

Isra University Hospital, Hyderabad

Email:drmurtazasameen@gmail.com

Received: August 16, 2020

Accepted: September 2, 2020 syndrome according to American Uveitis society diagnostic criteria. ${ }^{2}$

This syndrome consists of four clinical stages: a) in first stage there are nonspecific symptoms such as nausea, fever and the neurological symptoms like headaches, muscle weakness; 2) ophthalmologic stage, in which patients complain of blurred vision, pain, photophobia or central scotoma (bilateral in $80 \%$ of the cases); bilateral serous retinal detachment often occurs, tinnitus is often present; 3 ) third stage, occurs within months from the onset and is characterized by poliosis involving the eyebrows, eyelashes, hair loss and vitiligo; 4) chronic recurrent stage: recurrent uveitis and ophthalmological complications. ${ }^{3}$

The aim to report this case is to familiarize ophthalmologists and health care professionals about its findings and complications that are usually found in such patients. 


\section{CASE PRESENTATION}

A well oriented 37-year old female presented to Isra University hospital on August 11, 2020 with complaint of chronic progressive deterioration of vision in left eye for 06 months and decreased vision in both eyes for 5 years. She was using Prednesone acetate eye drops and Atropine 1\% eye drops the for the last 3 years. On Ocular examination her best-corrected visual acuity (BCVA) was 6/60 (OD) and perception of light (OS), while the intraocular pressure (IOP) was $16 \mathrm{~mm}$

$\mathrm{Hg}$ (OD) and $18 \mathrm{~mm} \mathrm{Hg}(\mathrm{OS})$ measured with Goldmann applanation tonometer. The slit-lamp examination of right anterior segment showed diffuse Keratic precipitates along with diffuse iris atrophy and seclusio pupillae (360 degree). There were mild lenticular changes in right eye along with sunset glow fundus. Left eye revealed poliosis, diffuse Keratic precipitates, diffuse iris atrophy along with seclusio pupillae and white cataract. The fundus view of left eye was hazy due to cataract. Ultrasound B-Scan of both eyes was within normal limits.

On general physical examination a young female well oriented in time place and person showed numerous white patches throughout the body. She gave history of vitiligo which was diagnosed 2 years back at Isra university hospital. Meanwhile, she was also suffering from tinnitus. For this reason she was referred to E.N.T specialist for expert opinion. There was no significant history of trauma or neurological illness.

A cataract surgery was planned in her left eye

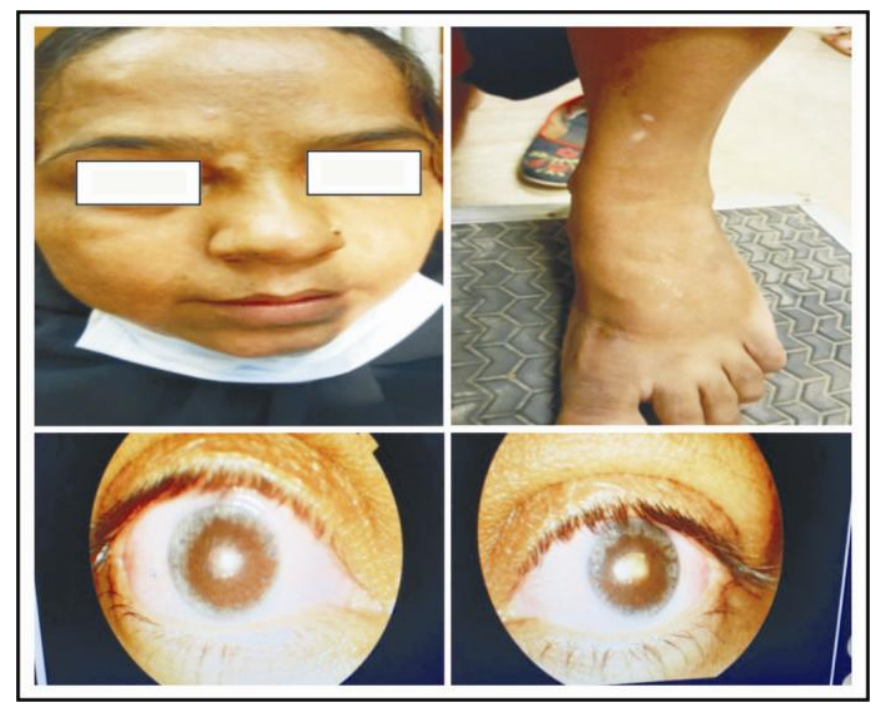

Fig. 1: 37 years old female with Vitiligo. Both eyes show 360 Posterior synechia, Iritis and Lenticular changes. at least 3 months after the absence of any ocular inflammation.

\section{DISCUSSION}

VKH syndrome is a rare multisystem autoimmune disorder in which the incidence and risk have not been substantially established. However ocular complications are adverse and can lead to vision threatening events. ${ }^{4}$ Uveitis is relatively common with the incidence of 0.3 to $1.2 \% .^{5}$ VKHD is an anti-melanocyte granulomatous autoimmune disease that affects eyes, inner ear, meninges, skin, and hair., It has been studied that HLA-DR4 is strongly associated with VKHD. The clinical features of VKHD include: bilateral chronic iritis, pan uveitis, neurological signs like headache, meningitis, and cutaneous findings of alopecia, poliosis, or vitiligo. ${ }^{7}$ Among individuals of pigmented skin including Asians, it is an important cause of noninfectious uveitis. ${ }^{7,8}$ Our patient was kept on topical steroids and cycloplegics which did not show improvement. The patient we reported here was a young female with an ocular and cutaneous involvement. There was no neurological involvement. Various immunesuppressants have been used to treat this disorder, however the risk of ocular complications remains, therefore regular follow-up is required.

In summary, although VKH is a rare disorder, the ocular complications can be vision threatening, therefore, a regular and long-term follow-up is necessary by an Ophthalmologist.

\section{Conflict of Interest}

Authors declared no conflict of interest.

\section{REFERENCES}

1. Neves A, Cardoso A, Almeida M, Campos J, Campos A, Castro Sousa JP. Castro Sousa Unilateral Vogt-Koyanagi-Harada Disease: Case Rep Ophthalmol. 2015; 6: 361-365.

2. Sakata VM, da Silva FT, Hirata CE, de Carvalho JF, Yamamoto JH. Diagnosis and classification of Vogt-Koyanagi-Harada disease. Autoimmun Rev. 2014; 13: 550-555.

3. Kurono Y, Takeda T, Kunimatsu Y, Tani N, Hashimoto N, Hirose K. Vogt-Koyanagi-Harada disease during chemo-immunotherapy for non-small cell lung cancer. Respirol Case Report, 2020; 8 (3): e00545. Doi: $10.1002 / \mathrm{rcr} 2.545$. 
4. O'Keefe GAD, Rao NA. Vogt-Koyanagi-Harada disease. Surv Ophthalmol. 2017; 62 (1): 1-25. Doi: 10.1016.

5. Street D, Sivaguru A, Sreekantam S, Mollan SP. Vogt-Koyanagi-Harada disease. Pract Neurol. 2019; 19 (4): 364-367. Doi: 10.1136.

6. Khan F, Zahid S, Raza SS, Iqbal M. A case of Vogt Koyanagi Harada disease in a 16 year old girl. JPMA. 2017; 67 (11): 1759-1761.

7. Chee SP, Jap A, Bacsal K. Prognostic factors of VogtKoyanagiHarada disease in Singapore. Am J Ophthalmol. 2009; 147: 15461.e1.

8. Cunningham ET, Rathinam SR, Tugal Tutkun I, Muccioli C, Zierhut M. Vogt Koyanagi Harada disease Ocul Immunol Inflamm. 2014; 22: 249-252.

\section{Authors' Designation and Contribution}

Rebecca; Resident: Design, Literature Research, Statistical Analysis Manuscript Editing.

Murtaza Sameen Junejo; Senior Registrar: Data Collection and Manuscript Writing.

Fahad Feroz Shaikh; Associate Professor: Data Collection and Manuscript Writing.

Nazir Ashraf Laghari; Professor: Manuscript Review and Final Approval of Manuscript. 